\title{
O.S.P.
}

L'orientation scolaire et professionnelle

$47 / 1 \mid 2018$

L'héritage de Maurice Reuchlin

\section{Reuchlin et la méthodologie}

Reuchlin and methodology

Jacques Juhel

\section{OpenEdition}

Journals

Édition électronique

URL : http://journals.openedition.org/osp/5600

DOI : $10.4000 /$ osp. 5600

ISSN : 2104-3795

Éditeur

Institut national d'étude du travail et d'orientation professionnelle (INETOP)

Édition imprimée

Date de publication : 15 mars 2018

ISSN : 0249-6739

Référence électronique

Jacques Juhel, « Reuchlin et la méthodologie », L'orientation scolaire et professionnelle [En ligne], 47/1 |

2018, mis en ligne le 01 mars 2020, consulté le 16 décembre 2020. URL : http://

journals.openedition.org/osp/5600; DOI : https://doi.org/10.4000/osp.5600

Ce document a été généré automatiquement le 16 décembre 2020.

(c) Tous droits réservés 


\title{
Reuchlin et la méthodologie
}

\author{
Reuchlin and methodology
}

Jacques Juhel

1 Les premiers travaux méthodologiques et techniques de Reuchlin - il était alors un jeune chercheur au CNRS - sont consacrés au domaine de recherche que Piéron lui assigne en priorité, c'est-à-dire à la méthode des tests, influente à l'époque en orientation et qui repose sur une théorie de l'attribut psychologique du sujet. Le premier article de Reuchlin, intitulé «Les qualités d'un bon test », est publié en 1947. Il tire son propos d'une étude, prise comme modèle, publiée en 1930 par Paterson et ses collaborateurs sur le test d'aptitude mécanique de Minnesota. Ce texte est bientôt suivi d'une quinzaine d'autres sur la validation des tests, leur pondération au sein d'une batterie, les procédés d'étalonnage ou l'observation du comportement. L'exigence méthodologique, l'acuité de l'esprit critique et la richesse prospective des vues y sont déjà remarquables. En voici une illustration.

2 Reuchlin (1948) présente une méthode d'observation et d'analyse des actes comportementaux ou « traits de comportement » de jeunes apprentis examinés à deux reprises avec les cubes de Kohs et les systèmes de leviers de Meili. Il observe que les distributions des traits ont une forme en J qui s'écarte de la distribution normale et constate que la constance des sujets dans leurs traits est faible et même parfois nulle. Il applique alors une analyse par groupement ${ }^{1}$ aux relations entre traits, démarche rare à l'époque, pour identifier les "modes d'activité " qui couvrent les actes comportementaux observés aux deux tests. Les résultats de cette analyse suggèrent l'existence de trois modes d'activité, non indépendants les uns des autres, interprétés en termes de rapidité, d'organisation et d'inorganisation. Après avoir pris soin de vérifier que la configuration proposée est acceptable en montrant que les corrélations entre traits de comportement prédites par ce schéma reproduisent suffisamment bien les corrélations observées, Reuchlin constate que ces trois modes d'activité «réapparaissent chez les mêmes individus lorsqu'ils accomplissent des tâches différentes" (Reuchlin, 1948, p. 147) et qu'ils permettent de prédire la réussite à l'atelier des apprentis. Il conclut que cette prévision strictement empirique apporte un élément de validité externe à la classification des traits de comportement qu'il propose. 
3 L'approche méthodologique adoptée dans cette étude suscite plusieurs remarques. L'observation, systématique et planifiée, y joue un premier rôle. La procédure est décrite avec précision, le mode de recueil des observations est guidé par des exigences d'objectivité, notamment par la confrontation des observations effectuées par deux observateurs indépendants. L'attention scrupuleuse que Reuchlin porte aux observations fait naître en lui des doutes sur certains présupposés de l'approche psychotechnique. Il estime ainsi, face à la non-normalité des distributions des actes comportementaux, qu'il n'y a aucune raison valable de considérer que ces observations présentent du point de vue de leur distribution «quelque analogie avec des variables biologiques distribuées normalement" (Reuchlin, 1948, p. 130). Il explique la faible constance des sujets dans leurs traits de comportement par l'idée que «subir un test pour la première fois est une situation foncièrement différente de celle qui consiste à le subir une deuxième fois » (Reuchlin, 1948, p. 136). Ces résultats inattendus l'amènent à s'interroger sur certains des postulats "nécessairement simplistes" qui fondent la méthode des tests, par exemple sur le choix de la loi normale comme modèle des échelles de mesure ou sur la fidélité comme "qualité » inhérente des tests. Mais, malgré ces difficultés qu'il considère comme « l'indice d'un certain déséquilibre dans la conception théorique sous-jacente " (Reuchlin, 1954, p. 316), les tests, en permettant « d'exprimer numériquement l'observation d'une phase de comportement » (Reuchlin, 1954, p. 320), restent pour lui des techniques d'observation plus «objectives » que beaucoup d'autres formes d'observation.

Dès ces premiers moments, la relation s'impose aux yeux de Reuchlin «comme une réalité plus stable que les données qui la matérialisent dans telle circonstance particulière " (1948, p. 138). La relation commune à des observations apparemment différentes et répétables peut donc être employée pour décrire le contenu d'une classe d'objets. Parce que les problèmes de la psychologie différentielle sont « concrets », que la science lui fournit des raisons de se méfier de l'expérience immédiate, mais qu'il veut rester dans le domaine d'une "psychologie du vérifiable " qu'il oppose parfois à une "psychologie du vécu», Reuchlin cherche à définir des objets relationnels pour produire une connaissance scientifique. Il insistera d'ailleurs très souvent dans son œuvre sur la nécessité de prendre appui sur des données de fait et des hypothèses vérifiables afin de "développer le corps des connaissances "objectives", c'est-à-dire "publiques", explicitement "transmissibles" et par là "cumulables" (1971, p. 61). La démarche de vérification qu'il adopte dans cette étude et qu'il déclare emprunter à Burt (1948, p. 145) possède ces qualités. Elle présente un caractère public, car les informations qui permettent de juger du bien-fondé de l'hypothèse de l'existence de modes d'activité différenciés ayant un "caractère relativement stable " (Burt, 1948, p. 150) sont mises à disposition de la collectivité. Il est intéressant de noter que cette démarche de vérification est l'ébauche, en l'absence de moyens techniques adaptés, de l'approche « confirmatoire » que les modèles d'équations structurelles populariseront quelque vingt-cinq ans plus tard.

On voit ici mise en œuvre, au niveau où il est possible d'en juger dans le contexte temporel et technique de cette étude, une approche méthodologique qui, si elle ne dispose pas des méthodes d'analyse adaptées, est largement filtrée par les préoccupations épistémologiques de son auteur. Reuchlin perçoit le caractère irréductiblement complexe des objets scientifiques soumis à l'étude de la psychologie différentielle. Il comprend que cette psychologie de terrain est plus concrète que la 
psychologie générale, mais qu'elle «a aussi bien des raisons de cultiver l'abstraction, la formalisation » (1965a, p. 295) : formalisation des observations, formalisation des hypothèses et formalisation des méthodes statistiques de traitement qui lui paraissent offrir les moyens d'investiguer et d'analyser les objets complexes étudiés en psychologie. Il mène alors une puissante réflexion épistémologique sur la «mesure » des grandeurs psychologiques ainsi que sur les fonctions des méthodes statistiques de traitement. Ces grands thèmes de réflexion constitueront l'objet de sa thèse d'État publiée sous l'intitulé « Les méthodes quantitatives en psychologie» (1962a).

\section{Quantification et mesure des différences individuelles}

6 La quantification est un signe d'objectivité dans la méthode des tests, mais les grandeurs psychologiques que cette méthode permet de définir sont-elles mesurables? À quelles conditions peut-on parler de mesure, quelle est la signification de cette opération en psychologie, et quel est son rôle dans la production des connaissances? Ces questions suscitent chez Reuchlin d'intenses réflexions épistémologiques dont il partagera le fruit dans plusieurs textes publiés au cours des années soixante (1962a, 1962b, 1963a, 1963b).

7 Reuchlin n'ignore pas que les mesures fournies par les tests ne satisfont pas les exigences logiques de la théorie représentationnelle de la mesure qui impose que toutes les propriétés des nombres trouvent des correspondants dans toutes les propriétés des choses. Si l'on souhaitait respecter ces exigences, écrit-il, «l'emploi du mot "mesure" deviendrait extrêmement rare en psychologie » (1962b, p. 25). Mais selon lui, une conception de la mesure réunissant en un même point de vue les positions représentationnelle et opérationnaliste peut être envisagée. Développée par Stevens, cette conception conduit à définir la mesure comme l'attribution de nombres aux observations psychologiques selon certaines règles plus ou moins restrictives. Le système des nombres n'est plus considéré «avec l'ensemble de ses propriétés, mais avec certaines d'entre elles seulement » (1962a, p. 51). La mise en correspondance entre les propriétés des nombres et les propriétés des observations psychologiques peut ainsi s'établir à différents niveaux (échelles nominales, ordinales, d'intervalles). Reuchlin, qui défend cette conception élargie de la mesure, est conscient des difficultés que soulève le choix d'une opération de mesure. Il souligne ainsi que le choix d'« un certain niveau [de partition des observations en classes] n'est en général pas unique » (1962b, p. 26), car plusieurs opérations empiriques peuvent être mises en correspondance avec la même relation formelle. Ce point est important, car la détermination quantitative d'une dimension en psychologie différentielle s'effectue en partitionnant des objets, « à une certaine échelle d'approximation» (1965b, p. 185), en une série de classes par rapport auxquelles les individus sont ordonnés de façon univoque et peuvent être différenciés. Les dimensions utilisées par le psychologue dépendent donc des critères par lesquels on les définit (1962a, p. 107 et suiv.) et du choix d'une opération de mesure qui s'effectue dans un contexte expérimental donné et avec des objectifs fixés.

8 La représentation des propriétés empiriques nécessite donc de la part du psychologue qu'il explique la nature des décisions prises, qu'il formule explicitement les propriétés des opérations de mesure et qu'il apporte des précisions sur les instruments qui définissent les objets mesurés dans le cadre expérimental. Elle nécessite aussi, nous dit Reuchlin, d'expliciter l'usage envisagé des mesures effectuées dans le cadre de 
l'expérience. Cette importance accordée à l'utilisation des mesures témoigne d'une préoccupation pragmatique soucieuse de déterminer ce qui fonde l'opération de mesure. Reuchlin rejette l'exigence méthodologique suivant laquelle «avant de mesurer, il faut savoir si ce que l'on mesure existe et ce que c'est» (1962a, p. 168). L'opération de mesure, du seul point de vue de sa cohérence interne, est indéterminée : elle ne peut se définir qu'en fonction d'un " amont " (l'objet de l'expérience et ce que le psychologue connaît a priori des conditions expérimentales) et d'un "aval» (les fonctions assignées à la mesure), et « cette intrication de contraintes antérieures et postérieures ne définit elle-même qu'un moment de la démarche du psychologue " (1963a, p. 218). La mesure n'est donc pas une question qui se pose a priori; elle n'est qu'une phase d'«un véritable processus d'approximations successives qui s'établit entre la définition du procédé de mesure et la définition de l'objet que l'expérience poursuit » (1962a, p. 169). Elle prend place dans une démarche qui se déroule dans une sorte de «mouvement continu de restructuration » (1963b, p. 220), dont le résultat est de préciser l'objet de la démarche. Ce positionnement épistémologique conduit Reuchlin à penser que les théories de la mesure, si elles offrent une axiomatique adaptée aux propriétés des données empiriques qui permet l'emploi de méthodes statistiques de traitement, "n'apportent rien par elles-mêmes» (1962a, p. 65). Il formulera trente-cinq ans plus tard un point de vue assez semblable à propos des modèles de réponse à l'item ${ }^{2}$ en écrivant qu' «il est possible que des psychologues puissent, dans certains cas, utiliser les résultats de leurs travaux» (1997, p. 235). L'intérêt de ces modèles psychométriques ne se justifie donc aux yeux de Reuchlin que si les possibilités de formalisation et de traitement qu'ils offrent permettent de répondre à un problème psychologique auquel les possibilités de formalisation et de traitement offertes jusqu'alors n'ont pas permis de répondre.

9 Le choix d'une opération de mesure dont Reuchlin pense qu'il n'est pas indépendant de l'utilisation des mesures, entraîne aussi une certaine définition de l'objet de la mesure et lui confère une signification particulière, un changement de l'opération de mesure pouvant entraîner un changement de définition et un changement d'interprétation de l'objet. L'analyse et les réflexions que Reuchlin conduit sur cette question fondamentale de la définition et de l'interprétation des objets mesurés, notamment à propos de la notion de dimension en psychologie différentielle (1963b), l'amènent à défendre un point de vue qui tente de rapprocher deux perspectives: la perspective opérationnaliste de Bridgman et la perspective de validation de construit ou «hypothéticodéductive » (1963b, p. 81) de Cronbach et Meehl, énoncée au milieu des années cinquante. On peut tenter de résumer comment Reuchlin conçoit ce rapprochement.

10 Dans son approche opérationnaliste, la mesure permet de constater une certaine forme d'organisation définie à partir de relations répétables entre observations, par exemple un ensemble de corrélations entre tests. Cette forme d'organisation échappe à l'expérience immédiate et peut varier selon le niveau de l'opération de mesure. Elle peut aussi se "réaliser ", écrit Reuchlin, par des opérationnalisations différentes d'une même notion; elle a donc un caractère relatif. Cette "intersection de relations répétables» (1962a, p.170) constitue le critère d'existence d'un phénomène, par exemple le pattern de corrélations positives observé par Spearman entre épreuves scolaires et tâches de discrimination. Le critère d'existence de l'objet ou de la classe d'objets repose donc sur un constat, une description, en même temps qu'il «apparait comme une explicitation» (1962a, p.99). Une définition opérationnelle est alors 
appliquée au phénomène sous la forme d'« un mot traduit par des "opérations" de mesure ou d'observation qui lui donnent un contenu observable, "empirique" » (1992, p. 33). Le mot qui désigne la variable opérationnelle peut être le même qu'un mot du langage non expérimental ou commun, mais parce qu'il lui est impossible de lever sa propre indétermination, ce mot du langage commun «fixe la pensée dans l'évidence » quand «une définition conventionnelle la libère» (1962a, p.113). La définition opérationnelle de la dimension permet alors d'étudier expérimentalement ses relations avec d'autres variables opérationnelles et de rechercher si le "contenu additionnel » (1963b, p. 82) mis éventuellement à jour et qui n'était pas impliqué par la définition opérationnelle permet d'en préciser la signification.

11 Dans la conception hypothético-déductive dont plusieurs aspects importants, note Reuchlin, auraient pu être plus développés qu'ils ne l'ont été par leurs auteurs (1963b, p. 88), il n'est pas nécessaire que les critères d'existence de l'attribut hypothétique qu'on prétend mesurer soient définis antérieurement au processus de validation. L'attitude méthodologique consiste à engager un ensemble de démarches hypothéticodéductives qui amènent à préciser ou modifier, en fonction des observations effectuées sur le réseau de lois, la théorie, qui inclut la dimension, la dimension que l'énoncé du réseau de lois permet de définir et l'opération de mesure. Dans ce réseau interprétatif que Reuchlin semble déjà percevoir comme un ensemble de variables en relation d'interdépendance réciproque dont la structuration peut être plus ou moins précisée, toutes les variables peuvent contribuer de la même façon à définir et interpréter le construit au même titre que l'opération de mesure peut servir à le faire. La distinction entre prédicteurs et critères s'estompe alors dans cette démarche réversible, les uns et les autres contribuant "à définir la dimension hypothétique sous-jacente pouvant rendre compte de leur corrélation » (1963b, p. 88). Les lois de ce réseau peuvent donc concerner des relations entre des variables observables, mais aussi des relations entre des variables observables et des variables hypothétiques comme celles que peuvent constituer les facteurs théoriques que l'analyse factorielle, une méthode formalisée de description des différences individuelles, permet de définir.

\section{Méthodes formalisées « et donc scientifiquement utilisables » de description des différences individuelles}

\section{Modèles et modes d'utilisation de l'analyse factorielle}

$\mathrm{Au}$ début des années cinquante, les progrès de l'analyse factorielle suivent l'évolution des idées en sciences humaines et sociales, et la méthode qui permet d'exprimer les relations entre tests sous une forme condensée et plus abstraite y trouve de larges applications. Reuchlin voit dans la perspective structurale de l'analyse factorielle « une sorte de moyen terme pouvant concilier dans une certaine mesure les exigences de la pratique et celles de la recherche » $(1995$, p. 23). Il apprend l'analyse factorielle, écrit-il (1988, p. 21), en lisant les ouvrages de Spearman, Burt et Thurstone sur l'analyse factorielle en facteurs communs et uniques. Il acquiert rapidement une grande expertise méthodologique et technique dans ce domaine auquel il consacrera de nombreuses publications tout au long de sa carrière, notamment au cours des années cinquante et soixante, fournissant encore en 2003 une contribution historique 
sur l'analyse factorielle et sur son évolution. Il publie en 1951 une étude employant cette méthode et en présente deux ans plus tard (1953a) les différentes formes : analyse factorielle entre variables, la plus employée, mais aussi analyse factorielle entre individus (méthode dite obverse) ou entre moments. En 1955, la responsabilité lui est confiée de rédiger le rapport de synthèse du colloque international du CNRS ayant pour thème "L'analyse factorielle et ses applications », ce qui traduit la reconnaissance de son expertise dans ce domaine. Il partage ses connaissances par la publication, une dizaine d'années plus tard, d'un ouvrage de référence intitulé Méthodes d'analyse factorielle à l'usage des psychologues (1964) qui s'impose à l'époque comme un ouvrage de référence. Les fondements théoriques et les hypothèses fondamentales de l'analyse factorielle y sont présentés; les différentes méthodes et leurs procédures de calcul y sont détaillées; les possibilités d'utilisation et les limites à l'intérieur desquelles l'analyse factorielle peut contribuer à l'accroissement des connaissances en psychologie y sont discutées.

13 L'analyse factorielle est une méthode de condensation d'un ensemble d'informations qui, sous sa forme la plus employée, est appliquée aux corrélations entre variables, par exemple des tests d'intelligence ou d'aptitude. Elle repose sur l'idée que le comportement individuel est le résultat de plusieurs influences qui peuvent être d'éventuelles « particularités constitutionnelles (aptitudes) » (1954, p. 311) ou, dans une perspective réciproque qui paraît tout autant légitime à Reuchlin et qui mérite d'être rappelée, des "appartenances à plusieurs groupes sociaux différents inégalement cohérents, inégalement "déterminants" " (1954, p. 317). L'analyse factorielle consiste à regrouper les tests d'après les classements des individus, les tests rangeant les individus à peu près de la même façon étant rangés dans une même classe. Chaque classe de tests est appelée un facteur. À chaque test, au sein de chaque classe, correspond une note en facteur du test, appelée saturation, qui est une estimation du degré d'appartenance du test à la classe. Formellement, l'observation effectuée chez "le sujet en situation" (1965b, p.184) est définie comme la somme de termes indépendants dont chacun est le produit de la saturation du test par le score factoriel de l'individu. Techniquement, le théorème fondamental de l'analyse factorielle établit que la matrice des corrélations est égale au produit de deux matrices inconnues (la matrice des saturations des tests sur les facteurs et sa transposée, obtenue en échangeant les lignes et les colonnes de même indice), dont l'analyse permet d'estimer les éléments pour un nombre de facteurs donné. En conséquence, il existe une infinité de solutions factorielles pour une même matrice de corrélations, problème dit d'«indétermination" des solutions factorielles. Les scores factoriels des individus peuvent être ensuite prédits, au besoin, à partir des estimations de la solution factorielle retenue et diffèrent donc avec celle-ci.

14 L'utilisation de l'analyse factorielle peut être envisagée de plusieurs façons. Elle peut être employée pour représenter sous une forme plus schématique des corrélations entre variables. Elle peut être aussi utilisée pour identifier par une démarche inductive ou découvrir par un raisonnement abductif une organisation des facteurs pouvant susciter a posteriori des hypothèses explicatives. Elle permet également d'éprouver une hypothèse, par exemple un modèle factoriel dont certaines caractéristiques ont été explicitées a priori. La conception de la «nature » des facteurs de l'analyse factorielle et leur caractère heuristique diffèrent selon l'orientation adoptée. 
15 La question de la nature des facteurs n'est pas pour Reuchlin celle du caractère concret de leur réalité. Il estime en effet que le contenu de la notion de facteur varie avec les variables et avec les individus, mais aussi avec le rôle que le psychologue confère aux facteurs dans le problème qu'il se pose, avec le genre de services que ces facteurs peuvent lui rendre au cours de la recherche et, « pour une part au moins, avec les idées admises dans le domaine d'utilisation envisagé » (1955b, p. 398). La question de la nature des facteurs est pour Reuchlin celle du principe qui a servi à guider leur identification ou leur définition. Or les chercheurs qui veulent identifier les facteurs nécessaires pour rendre compte des corrélations entre tests tendent souvent à considérer que les facteurs sont «l'indice de la présence d'unités fonctionnelles distinctes, dont chacune serait responsable du succès ou de l'échec dans une catégorie de tâches» (1964, p. 70): les "facteurs de l'esprit» pour Burt ou «les aptitudes mentales primaires » pour Thurstone. Reuchlin doute pour sa part de la possibilité de dresser un tel tableau d'aptitudes. Il est convaincu que les facteurs extraits de batteries de tests mentaux ne peuvent être assimilés à des aptitudes, car il n'y a pour lui " aucune raison d'attendre que l'analyse factorielle atteigne nécessairement des entités psychologiques dont la stabilité garantirait le caractère fondamental et universel » (1964, p. 70). Il juge aussi trop étroite la correspondance faite entre tâche et aptitude, car «les résultats de l'analyse factorielle ne sont pas uniques, comme ils devraient l'être s'ils reflétaient la présence d'unités fonctionnelles » (1954, p. 313).

Il faut peut-être rappeler, pour mieux comprendre les raisons qui conduisent Reuchlin à défendre ce point de vue, l'importance théorique que celui-ci accorde à la distinction entre la méthode d'analyse en composantes principales et les méthodes d'analyse en facteurs communs et facteurs uniques (méthodes centroïdes, méthodes fondées sur le principe du maximum de vraisemblance) (1964). On sait que l'analyse en composantes principales consiste à analyser entièrement la variance des tests employés et qu'elle permet de reconstruire les observations de manière unique et avec un nombre de facteurs égal au nombre de tests. Les méthodes d'analyse en facteurs communs et facteurs uniques veulent reconstruire la structure des corrélations entre tests; c'est l'intérêt majeur qu'elles présentent aux yeux de Reuchlin. Elles portent donc seulement sur la variance commune aux tests, sur leurs communautés et nécessitent un nombre de facteurs (communs et spécifiques) plus élevé que le nombre de tests. Ces méthodes conduisent donc à une indétermination des solutions factorielles qui résulte de plusieurs sources d'indétermination. Elles sont, dès leurs premiers développements et jusqu'alors, l'objet de réserves de la part de certains mathématiciens et de controverses comme celle avec Benzécri, l'inventeur de l'analyse des données «à la française ».

17 Reuchlin estime que l'indétermination des solutions factorielles ne pose pas problème en soi si l'analyse factorielle est employée par le psychologue pour définir des facteurs plutôt que pour les identifier, si ces facteurs sont le moyen d'éprouver une hypothèse plutôt que s'ils sont considérés comme la cause commune aux corrélations entre tests (1964, p. 52 et suiv.). L'indétermination peut être levée de plusieurs façons (1955a). Elle peut l'être en définissant des critères mathématiques qui permettent de trouver des "facteurs observés ", c'est-à-dire des facteurs qui «ne dépendent pas de l'analyste, mais des données » (1955a, p. 187). Un critère formel de ce type peut être par exemple de décider qu'un facteur doit rendre compte du maximum de variance des données observées. Mais, estime Reuchlin, ce critère de simplicité formelle n'a pas nécessairement une valeur heuristique sur le plan psychologique, et un critère de 
contenu cohérent et interprétable peut lui être préféré. L'indétermination peut alors être levée sur la base d'une théorie psychologique et de connaissances antérieures relatives au domaine considéré qui permettent de choisir, "pour des raisons extérieures aux données elles-mêmes » (1955a, p. 205), une certaine solution factorielle compatible avec une hypothèse plausible relative à un certain domaine : c'est l'objet des méthodes de rotation. L'hypothèse peut être très générale, comme la recherche d'une structure de forme identique dans des analyses différentes, par exemple la "structure simple» de Thurstone. Mais d'autres critères théoriques plus particuliers peuvent guider la rotation comme la recherche d'une discrimination maximale entre deux groupes de variables, objectif pour lequel Reuchlin et Bacher (1957) développent une méthode de rotations discriminantes qui utilise les fonctions discriminantes de Fisher. "facteurs théoriques [...] dont l'intérêt est éventuellement démontré par l'utilité " (1955a, p. 208), c'est-à-dire par les services théoriques ou pratiques qu'ils peuvent rendre au psychologue. Ainsi,

«des théories différentes peuvent évidemment être utilisées, et la solution factorielle ne sera qu'une modalité d'expression de la théorie choisie. Plusieurs théories peuvent conduire à adopter la même solution. Malgré leur identité matérielle, il nous semble que ces solutions n'ont pas alors la même signification, qu'elles n'appellent pas les mêmes vérifications, qu'on ne peut en tirer les mêmes conséquences » (1955a, p. 193).

Il en est ainsi des solutions factorielles de Burt et de Thurstone, même si les divergences entre solutions factorielles peuvent aussi disparaître, comme Reuchlin le démontre (1955a, table 14, p. 203), en introduisant certains principes théoriques. Ces principes appliqués à l'étude des corrélations entre tests mentaux permettent l'expression des facteurs cognitifs sous une forme hiérarchique qui comporte un facteur général et des facteurs de groupe, une hiérarchie de niveaux pouvant évoquer, assez pauvrement dira Reuchlin plus tard $(1997$, p. $105 ; 1998$, p. 103), une organisation mentale.

Reuchlin envisage donc deux modes d'utilisation de l'analyse factorielle. Le premier est exploratoire: son objectif est de décrire et, éventuellement, de trouver des groupements de variables à partir d'un échantillon de variables du domaine étudié. Le second est hypothético-déductif: l'analyse factorielle est «intégrée à l'analyse expérimentale " et "organisée en vue de la vérification d'une hypothèse précise " (1955b, p. 400). Elle porte sur un échantillon systématique de variables et s'appuie sur l'exactitude de la reconstruction empirique pour juger si l'hypothèse est corroborée, ou pas, par les faits. Pour Reuchlin, seuls les facteurs définis a priori et fournis par ce second mode d'utilisation peuvent constituer des variables théoriques que l'inclusion dans un réseau de lois permet de mieux définir.

\section{L'analyse de variance comme méthode de description analytique des données}

21 Parallèlement aux recherches qu'il effectue sur l'analyse factorielle au début des années cinquante, Reuchlin s'intéresse à l'analyse de la variance à plusieurs facteurs de classification développée par Fisher au début des années vingt. Le modèle d'analyse de la variance fournit une représentation des données recueillies sur des groupes 
constitués selon un plan d'expérience donné. La variable dépendante continue est définie comme une somme de plusieurs termes: sa moyenne générale, plusieurs moyennes hypothétiques qui représentent les contributions des sources théoriques de variations contrôlées expérimentalement et l'erreur dont la variance doit être estimée pour tester les comparaisons effectuées. En permettant de faire varier simultanément plusieurs facteurs de classification (entre conditions expérimentales ou entre groupes de sujets) et de tester des hypothèses correspondant chacune à une source particulière de variations, l'analyse de la variance ouvre donc des possibilités nouvelles à l'analyse expérimentale. Deux ans après l'introduction par Faverge de l'analyse de la variance auprès des psychologues de langue française, Reuchlin publie un texte sur les plans d'expérience fishériens et sur la logique qui en sous-tend l'application (1953b).

Les principes de Fisher et leur formalisation constituent un apport essentiel aux yeux de Reuchlin qui y voit "une sorte d'axiomatisation de la démarche de l'expérimentateur» (1965a, p. 300). Cette axiomatisation fonde la signification de l'expérience sur la correspondance entre le plan expérimental, la forme du modèle statistique employé, "aspects différents d'un même tout» (1953b, p.61), et la démarche logique d'interprétation. Elle oblige à s'appuyer sur une formalisation rigoureuse avant de recueillir et d'analyser des observations concrètes, à remplacer l'intuition par un raisonnement basé sur des critères formels. Elle donne une grande cohérence à l'étude des influences simultanées qui s'exercent à chaque instant sur chaque individu.

Reuchlin reconnaît de nombreux mérites aux enseignements de Fisher. L'analyse de la variance à plusieurs facteurs de classification offre une description « économique » des données dans le sens où une partie des variations de la variable dépendante est attribuable à un nombre limité d'effets systématiques des variables indépendantes. Elle permet aussi d'estimer les effets d'interaction, c'est-à-dire les effets des variations concomitantes de plusieurs variables indépendantes, orthogonales par hypothèse. La notion d'interaction a une portée générale, car c'est à son niveau «que peuvent être décrites les relations intriquées de plusieurs variables dont le jeu complexe constitue le plus souvent l'objet même de la psychologie » (1962a, p. 239). Reuchlin souligne aussi l'importance de la réciprocité de l'interaction. Le raisonnement à propos d'une interaction peut en effet être appliqué dans plusieurs sens, par exemple dans une interaction simple, à n'importe laquelle des variables par rapport à la relation entre les deux autres. En permettant cette réciprocité que l'on retrouve avec la notion de corrélation partielle entre deux variables, calculée en maintenant constante une troisième, l'interaction préserve ainsi de la tentation "d'une imputation causale hâtive » (1962a, p. 233) à laquelle Reuchlin invite à ne pas céder. La notion d'interaction rapproche aussi avec sa réciprocité des méthodes multivariées dans lesquelles les données recueillies avec un ensemble de variables administrées à un ensemble d'individus sont considérées simultanément, le statut conféré par l'analyse à ces variables (indépendantes ou dépendantes) pouvant changer selon le sens de la chaîne de « causalité » envisagée.

Reuchlin formule néanmoins des réserves sur le sens et des objections sur l'usage des épreuves de signification dans le modèle d'analyse de la variance. Il s'interroge sur la capacité des modèles dans lesquels l'effet d'une variable indépendante sur une variable dépendante est estimé ceteris paribus, à décrire et à expliquer les différences individuelles. La perspective interactionniste dans laquelle il s'inscrit le fait douter de 
la pertinence d'une conception qui réduit l'étude d'un phénomène psychologique observé chez "le sujet en situation" (1965b, p. 184) à un ensemble d'imputations causales élémentaires. L'invocation sur le modèle du fonctionnement cérébral ou cognitif d'une structure, d'un réseau d'unités d'analyse entretenant des relations fonctionnelles les unes avec les autres, lui paraît plus appropriée pour accroître l'intelligibilité du phénomène étudié.

\section{Les interrogations sur la méthodologie analytique en psychologie différentielle}

Dès ses premiers travaux sur l'analyse de la variance, Reuchlin (1953b) signale les problèmes de signification que posent les comparaisons de groupes sur la base d'une estimation statistique de l'erreur, le caractère peu vraisemblable en psychologie de l'hypothèse nulle, la dépendance de la signification statistique à un seuil arbitrairement choisi et à l'effectif de l'étude, ou la nécessité de distinguer la signification statistique et la signification pratique. Il reviendra d'ailleurs fréquemment sur les problèmes d'ordre statistique et technique que posent les épreuves de signification (1962a, 1971, 1976, 1977) ainsi que sur les limites de l'inférence statistique dans une conception de la recherche qui veut accroître l'intelligibilité d'un phénomène plutôt qu'identifier les lois qui le gouverneraient $(1993,1995,1997,1998)$.

Rappelons que dans une épreuve de signification statistique appliquée à une différence de paramètres (e.g. entre deux moyennes observées), celle-ci est expliquée en posant deux hypothèses concurrentes: l'une statistique, l'autre psychologique. Dans l'inférence statistique fréquentiste, le statisticien fait l'hypothèse d'une différence nulle entre les paramètres et en dérive une distribution d'échantillonnage des valeurs de la différence observée. Mais ce modèle, écrit Reuchlin, «constitue une procédure permettant d'élaborer de façon précise une réponse à une question que le psychologue ne se pose pas» (1977, p. 282) car la structure du modèle du psychologue n'est pas suffisamment formalisée pour tester cette hypothèse nulle. Ainsi, explique-t-il, des valeurs proches mais non significativement différentes de 0 peuvent témoigner d'effets intéressants au plan théorique quand des valeurs proches mais significativement différentes de 0 peuvent ne pas justifier l'utilité d'une intervention par le praticien. De plus, l'identification dans l'épreuve de signification de la population de référence est incertaine, des éléments de jugement subjectif entrent dans le choix des seuils de signification (risques de types I et II), le degré de significativité de l'effet est souvent confondu avec sa taille et l'accroissement du nombre d'observations augmente la probabilité de rejet de l'hypothèse nulle. Ces critiques radicales à l'égard de l'inférence fréquentiste feront d'ailleurs regretter à Reuchlin que la tradition fishérienne ait «si étroitement orienté les travaux des psycho-statisticiens français » (2003, p. 26).

Développée au milieu des années soixante-dix par Rouanet et son équipe, l'inférence statistique fiducio-bayésienne apparaît à Reuchlin mieux adaptée aux besoins du psychologue. La démarche statistique consiste cette fois, à partir d'une valeur de la différence observée, à spécifier une distribution de probabilités a priori et à calculer au moyen du théorème de Bayes la distribution a posteriori des valeurs « crédibles » de la différence entre paramètres. Au contraire de l'approche fréquentiste, l'approche bayésienne permet donc de parier sur l'amplitude de la différence entre paramètres et de tester directement l'hypothèse de recherche. Elle impose bien sûr, comme 
l'approche fréquentiste, de faire certains choix, par exemple de définir la valeur à partir (resp., en dessous) de laquelle on considère que la différence est "notable " (resp., "négligeable») ou de préciser la probabilité associée aux valeurs de cette différence pour un niveau de garantie fiduciaire donné. Mais l'attitude méthodologique n'est pas du tout la même; elle oblige à relativiser, à « diversifier en fonction des conditions concrètes d'utilisation une règle (dogme, recette) dont le respect aveugle ne donne qu'une image caricaturale de ce qu'est la rigueur scientifique » (1996, p. 245). Mais malgré les avantages que voit Reuchlin à l'inférence bayésienne dont il présente les idées et souligne l'intérêt dans plusieurs textes (1977, 1992, 1998), l'une et l'autre de ces deux approches lui semblent néanmoins se heurter aux mêmes difficultés fondamentales (1998, p. 101 et suiv.). Le choix des critères dont dépend l'interprétation psychologique étant de la responsabilité du psychologue, aucune épreuve de signification formelle ne peut, écrit-il, «se substituer mécaniquement à son bon sens et à sa réflexion sur le sens de sa démarche » (1992, p. 76).

28 En soulignant les difficultés pour le psychologue de qualifier de significative ou de non significative une différence entre paramètres ou une corrélation, Reuchlin interroge la possibilité de vérifier empiriquement la prévision de modèles analytiques comme l'analyse de la variance ou l'analyse de régression multiple. Au scepticisme à l'égard des épreuves de signification s'ajoutent des doutes sur la capacité de ces méthodes à faire progresser l'étude des différences individuelles et à rendre service au psychologue travaillant sur le terrain. Reuchlin considère en effet que ces modèles ne peuvent comporter qu'un nombre réduit de variables quand il faudrait en disposer d'un grand nombre et que les plans d'expérience qui cherchent "à découvrir le ou les facteurs (variables indépendantes) dont les variations sont associées à celles du phénomène étudié (variables dépendantes) toutes choses par ailleurs étant fixées ou variant de façon aléatoire» (1992, p. 161) rendent mal compte de l'organisation complexe des relations entre ces variables. Confronté dans ses premiers travaux aux problèmes méthodologiques que posent certaines liaisons «inextricables» étudiées en psychologie différentielle, comme celles entre variables socio-économiques et variables «biométriques » auxquelles il a pu s'intéresser, Reuchlin se demandait d'ailleurs déjà " s'il n'y a pas une erreur foncière à rechercher quel peut être l'effet d'une variable quelconque, métier du père ou revenu par exemple, en s'efforçant de maintenir constantes les autres variables » $(1951$, p. 269$)$.

On peut comprendre, au seuil de la révolution informatique qui apportera les outils techniques que l'on connaît, qu'un certain pessimisme transparaisse chez Reuchlin, ce que plusieurs participants à un colloque du CNRS sur «Les modèles et la formalisation du comportement» (1967) ne manquent pas de lui faire remarquer. Reuchlin voit bien l'intérêt des modèles analytiques lorsqu'ils sont appliqués en laboratoire à des données décontextualisées ou artificielles mais, dit-il, « les difficultés apparaissent dans les cas où ce contexte ne peut être éliminé » (1971, p. 61). Il se demande si la structure de ces modèles analytiques n'est pas trop simple pour pouvoir rendre compte de la complexité des objets étudiés en psychologie différentielle et en psychologie sur le terrain. Le psychologue qui étudie les conduites dans leurs conditions habituelles est pourtant obligé, répond-il à Fraisse, « de chercher, dans les techniques qu'on connaît, celles qui s'adaptent le moins mal possible au problème posé » tandis que celui qui travaille en laboratoire peut toujours simplifier le problème pour réduire celui-ci au niveau technique qu'il emploie, avec peut-être le risque que le problème n'ait «finalement 
plus aucun rapport de nature avec celui que l'on se propose d'étudier» (1967, p. 377). Or, estime-t-il, peu de progrès ont été effectués dans le développement de modèles qui permettraient d'«étudier plus scientifiquement les différences individuelles » (1967, p. 381).

Ce pessimisme n'est que transitoire, car Reuchlin perçoit très tôt que l'analyse en pistes causales, présentée par Boudon sous le nom d'analyse de dépendance ${ }^{3}$ dans L'analyse mathématique des faits sociaux en 1967, peut être utilisée pour décrire les relations entre des variables considérées au sein du système qu'elles forment ensemble. Rappelons brièvement les grandes étapes des méthodes dites d'analyse causale. Des hypothèses qualitatives sont d'abord posées sur l'existence de certaines relations entre variables observées prises pour cause ou pour effet au sein d'une configuration structurelle donnée, ce qui nécessite une connaissance du contexte causal. Les effets des variables indépendantes sur les variables dépendantes, intermédiaires ou ultimes, sont ensuite estimés empiriquement sur la base d'assomptions statistiques (détermination séquentielle et non conjointe), ce qui permet d'infirmer ou d'accepter provisoirement le « schéma causal » postulé.

31 Reuchlin, dans la brève exposition qu'il donne quelques années plus tard de ce qu'il appelle l'« épreuve formelle d'un schéma causal en matière de corrélations totales ou partielles» (1971, p. 72), exprime pourtant plusieurs réserves à l'égard de cette méthode appliquée à des variables qui différencieraient les individus dans des conditions habituelles de vie. La condition de récursivité qui implique que chaque variable-effet puisse être strictement déduite des variables prises pour cause lui semble peu réaliste au niveau d'observation qui est celui du psychologue. Elle est d'ailleurs incompatible avec l'idée de réversibilité à laquelle Reuchlin est très attaché et que la corrélation traduit parfaitement. De plus, le choix des variables susceptibles d'avoir un effet sur le phénomène que le schéma causal représente ne peut être effectué qu'en faisant appel à des connaissances relatives au problème posé dont certaines ne sont pas encore formalisées. Le psychologue n'est donc jamais formellement sûr d'avoir défini un système clos de variables, c'est-à-dire un système tel que chaque variable non incluse dans le système affecte au plus une variable du système.

Mais Reuchlin voit aussi plusieurs avantages à l'emploi de l'analyse causale. Les schémas de causalité postulés peuvent être complexes, laissant ainsi de côté le principe d'économie et de simplicité "incompatible avec le caractère organisé du vivant" (1995, p. 25). Le même schéma causal peut aussi être analysé de façons différentes, ce qui permet, comme dans l'analyse factorielle ou l'analyse de régression multiple, de choisir une démarche d'analyse ne se basant pas exclusivement sur des critères statistiques valant quel que soit le problème posé. Il remarque avec intérêt que « des modèles différents peuvent conduire aux mêmes prédictions » (1971, p. 73), ce qui rend nécessaire de faire intervenir des connaissances antérieures sur le domaine investigué qui seules permettent au psychologue de choisir entre différentes représentations également compatibles avec les faits. Malgré les limites de l'analyse en pistes causales et les critiques qu'il lui adresse, Reuchlin perçoit dans cette méthode la possibilité de mettre en œuvre une démarche qui procède par "approximations successives " et cherche à tester des hypothèses structurales dans l'étude des conduites considérées dans les conditions habituelles de vie. 


\section{De l'explication causale à l'explication structurale}

33 L'utilisation par Reuchlin de l'analyse factorielle pour modéliser des hypothèses structurales s'est toujours accompagnée de réserves à l'égard de l'explication causale, entendue dans son sens le plus strict. Les facteurs communs, écrit-il, ne possèdent pas " certains caractères que l'on attribue volontiers, en première analyse, à l'explication causale » (1962a, p. 362). La cause n'est pas antérieure à l'effet, et l'effet ne disparaît pas dès qu'est supprimée la cause, puisque celle-là est induite de l'effet. La relation n'a donc pas ici de caractère nécessaire. En outre, "l'imputation causale est en général impossible, de quelque façon que les résultats soient traités» (1962a, p.379), puisqu'une même corrélation peut suggérer au niveau de description qui est celui du psychologue plusieurs relations causales. La notion de "complexe causal» (1962a, p. 391), que discute Reuchlin, veut d'ailleurs marquer l'absence de cause ultime et de niveau ultime dans l'explication.

Cette remise en question de l'explication causale est le résultat de choix épistémologiques fondamentaux affirmés avec force à de nombreuses occasions par Reuchlin (e.g. 1962a, 1980, 1995). Il refuse ainsi la rupture épistémologique entre la description et l'explication des conduites considérées dans les conditions habituelles de vie et propose de considérer la description comme une explication d'un moindre niveau d'explicitation. Il conteste la pertinence pour la psychologie différentielle et la psychologie de terrain des critères de cohérence théorique et d'économie qui guident vers plus de simplicité formelle et conduisent souvent à une vision ascendante des déterminismes de la conduite. Il rejette l'hypothèse de singularité fonctionnelle selon laquelle le même effet a toujours la même cause et accorde au contraire une importance fondamentale à la signification fonctionnelle des situations naturelles «dans lesquelles ont lieu plusieurs processus d'élaboration de la réponse, processus pouvant se substituer l'un à l'autre" (1978, p.134). Ces choix épistémologiques conduisent Reuchlin à défendre une conception fonctionnaliste de la structuration des conduites dans laquelle « formuler une explication causale n'est plus définir la "raison d'être" des phénomènes " $(1980$, p. 247), mais construire un modèle intelligible par rapport auquel la notion de causalité devient relative. À la compréhension structurale initiale, qui bravait « l'interdit positiviste à l'égard de la recherche des causes » (1962a, p. 362) et voyait dans les facteurs communs des constructions rationnelles susceptibles de fournir un certain type d'explication causale, succède une conception qui cherche à mieux s'appliquer à l'explication d'un fonctionnement et donne plus d'importance aux fonctions qu'aux causes. L'émergence conjointe d'une nouvelle famille de méthodes statistiques de traitement, les modèles d'analyse des structures de covariance ou « modèles structuraux », laisse alors espérer à Reuchlin la possibilité de mettre en actes cette perspective structurale dans l'étude des différences individuelles.

Les modèles structuraux, développés au début des années soixante-dix par Jöreskog et introduits en France par Bacher (1984, 1987, 1988, 1989), intègrent en un même cadre méthodologique l'analyse en facteurs communs et uniques et l'analyse en pistes causales. Ils permettent de modéliser les relations entre des variables observées, entre des variables observées et des variables latentes (facteurs communs observés ou théoriques, variables théoriques d'erreur) ou entre des variables latentes. Leur grand intérêt réside dans la possibilité qu'ils offrent de tester des hypothèses structurales 
c'est-à-dire des hypothèses relatives à la structure d'ensemble des variables considérées.

L'étude des conduites en conditions habituelles de vie passe par l'observation systématique des propriétés d'un système de variables choisies sur labase des connaissances et des hypothèses psychologiques relatives au domaine considéré. L'examen des propriétés du système et de ce qu'elles impliquent du point de vue de l'organisation des relations entre variables conduit à la construction d'une représentation des relations entre les variables. Cette représentation est une sorte de schéma fonctionnel, c'est-à-dire la formalisation abstraite d'une structure en fonctionnement parmi d'autres envisageables. Une certaine connaissance du processus de génération des observations que les données statistiques seules ne suffisent pas à préciser est donc nécessaire pour atteindre une explication du phénomène qui tend à se ramener à l'interprétation de cette représentation structurale. Les relations fonctionnelles entre variables peuvent prendre plusieurs formes et se modifier en fonction du contexte que constituent les autres variables du système. Certaines, non déterminées a priori, sont de simples associations. D'autres, orientées par hypothèse, font intervenir plus ou moins explicitement la notion de variable-cause lorsque par exemple plusieurs variables sont supposées être les manifestations d'un même facteur ou qu'une variable est supposée avoir une influence sur une autre (relation récursive entre un antécédent et un conséquent). Ces relations peuvent avoir une autre forme qu'une relation cause-effet ponctuelle lorsqu'on suppose, par exemple, que deux variables entretiennent une relation réciproque non récurrente. Cette structure fonctionnelle guide l'étude du mécanisme générateur des données que l'évolution des techniques de modélisation statistique rend aujourd'hui possible. Si les assertions du modèle sont compatibles avec les données, le mécanisme empirique renforce la validité de la structure formelle. Le modèle explique, en un certain sens, dans la mesure où il rend intelligible l'organisation du réseau de relations qui détermine le phénomène que la structure englobe. Si les assertions du modèle ne sont pas corroborées par les faits, il faut alors modifier certains aspects de la structure ou en envisager une autre qui guidera l'étude du mécanisme de génération des données dont la compatibilité avec les données sera à nouveau évaluée dans une démarche d'approximations successives.

Cette manière d'envisager l'explication par un fonctionnement conduit à s'interroger sur le sens donné à la «notion de fonction ». Reuchlin (1990) évoque deux façons de comprendre cette notion, chacune d'entre elles faisant référence à des niveaux d'activité distincts. La «fonction» peut d'abord renvoyer aux lois qui régissent le déroulement de l'activité, à des mécanismes. Le mécanisme peut être restreint à un enchaînement de causes et d'effets ou être envisagé en référence à un système, c'est-àdire en matière d'organisation. Mais, au-delà de l'explication d'un phénomène par « la description du fonctionnement qui suscite son apparition [...] on peut franchir un pas de plus et observer que ce fonctionnement paraît s'expliquer par sa fonction, par le rôle qu'il paraît jouer dans l'adaptation » (1995, p. 277). La fonction fait alors référence à « la finalité apparente » $(1990$, p. 21) de l'activité de l'individu dans une situation donnée, à son orientation vers un objet-but prioritaire. Revenons brièvement sur chacune de ces conceptions.

38 La "fonction", dans sa première acception, est le rôle joué par une variable dans le fonctionnement du système, l'effet utile pour la structure au sein de laquelle la variable produit cet effet. Dans cette conception, expliquer un phénomène c'est identifier les 
fonctions, les rôles des variables dans la structure en fonctionnement et modéliser l'organisation des fonctions du mécanisme qui génère le phénomène. Cette conception mécaniste est celle que les modèles structuraux de type LISREL appliquent à la structure d'ensemble ainsi qu'aux variables psychologiques qui la composent ${ }^{4}$. Un des objectifs de la modélisation structurale est en effet de comprendre quelle est la fonction de ces variables en interprétant leur rôle en référence au fonctionnement de la structure. La détermination des fonctions des variables n'est pas causale (il ne s'agit pas d'un simple enchaînement de causes et d'effets), mais structurale, fonctionnelle. Ainsi, une même variable peut avoir un effet différent suivant l'état fonctionnel du système au sein duquel elle se trouve, et une même fonction d'ensemble peut être remplie par des structures ou des sous-structures différentes de variables. Reuchlin a présenté et discuté une recherche effectuée dans son laboratoire, qui formalise l'idée de vicariance processuelle chez des élèves en situation d'apprentissage en montrant que différentes combinaisons de variables-amont du système peuvent fournir la même valeur attendue de la variable-aval (1999, p. 174).

Dans la seconde conception, celle de la "fonction-but", il ne s'agit plus de tenter de comprendre la fonction d'un élément du point de vue de son effet, structuralement déterminé, sur le fonctionnement du système dont il fait partie. La fonction assumée par la structure en fonctionnement est de permettre l'atteinte d'un objet-but. La causalité paraît alors s'inverser, comme si l'effet sélectionné du fonctionnement (i.e. une certaine forme de réussite adaptative) pouvait expliquer la présence de la structure qui l'exerce. C'est à la finalité adaptative des fonctionnements, à cette conception téléologique de la fonction, que Reuchlin semble attacher une importance particulière lorsqu'il écrit que "le rôle évident du fonctionnement d'une structure est d'assurer une fonction, c'est-à-dire une contribution à une certaine forme d'adaptation » (1995, p. 24) ou que « la conduite a un but, un "propos", et c'est cette finalité qui va régir son organisation, lui conférer son unité » (1995, p. 59). On reconnaît ici un autre objectif, sans doute plus ambitieux, de la modélisation structurale qui est en relation première et nécessaire avec les connaissances et les hypothèses psychologiques sur le phénomène étudié : comprendre quelle est la fonction, dans un contexte donné, de la structure conceptuelle étudiée et à laquelle diverses structures empiriques peuvent être associées. Mais Reuchlin n'a pas fourni à notre connaissance d'illustration empirique permettant de juger de la portée théorique et de l'intérêt pratique de cette conception téléologique dans l'étude des différences individuelles. On peut imaginer qu'une recherche construite dans cette perspective pourrait par exemple s'appuyer sur la constitution de groupes d'individus fortement homogènes au regard de la perception qu'ont leurs membres de la situation qui leur est proposée et des objectifs poursuivis et sur la comparaison dans différentes situations et/ou à différentes occasions des représentations structurales compatibles avec l'organisation des données recueillies sur chaque groupe. Il y a là très certainement de nombreuses pistes de recherche intéressantes à explorer.

\section{Conclusion}

Nous avons tenté de dégager dans ce travail les aspects les plus caractéristiques de l'œuvre méthodologique de Reuchlin en illustrant le cheminement de sa pensée et son évolution vers les méthodologies et les modèles structuraux. Reuchlin a exercé sa 
réflexion sur la méthodologie psychologique en discutant les fondements épistémologiques de cette méthodologie. Le cœur de sa réflexion n'a pas été, comme le plus souvent aujourd'hui, d'évaluer l'efficience statistique des modèles statistiques de traitement des données mais de discuter leurs fondements épistémologiques, leur intérêt théorique et leur utilité pour le psychologue. De cette interaction entre options méthodologiques et réflexions épistémologiques, double domaine qu'il a " cultivé » tout au long de sa carrière, ont émergé de riches enseignements: les exigences méthodologiques d'objectivité, la nécessité du caractère public et répétable de la démarche, l'adhésion à une conception pragmatique de la mesure, l'orientation vers des représentations structurales de la conduite dans lesquelles les variations d'une variable prise pour effet ne peuvent être expliquées qu'à l'échelle d'une structure de variables observables et / ou hypothétiques, l'emploi de méthodologies structurales adaptées à la complexité des problèmes que se pose le psychologue, le souci constant de maintenir en relation réciproque les aspects techniques avec des considérations formelles, les connaissances formalisées ou intuitives du psychologue sur le contenu du domaine et son «bon sens».

Reuchlin a pris conscience avec une parfaite netteté de la spécificité du problème de l'analyse des différences individuelles en conditions habituelles de vie et de l'apport des méthodes formalisées à l'étude scientifique de ces différences. On ne trouvera sans doute pas de recette dans l'œuvre méthodologique qu'il nous a laissée sinon les marques d'une pensée à la fois facilitante, parce qu'elle pose souvent les questions que l'on ne sait pas (ou que l'on ne veut pas) se poser, encourageante, parce qu'elle offre les moyens conceptuels et méthodologiques d'y apporter des réponses provisoirement satisfaisantes mais aussi parfois déstabilisante, quand cette pensée toujours en évolution n'est déjà plus tout à fait là où on croyait l'avoir saisie.

\section{BIBLIOGRAPHIE}

Bacher, F. (1984). Les méthodes statistiques en psychologie différentielle : perspectives de développement. Psychologie française, 29, 9-15.

Bacher, F. (1987, 1988). Les modèles structuraux en psychologie. Présentation d'un modèle : LISREL. Première partie : Le Travail humain, 50, 347-370. Deuxième partie : Le Travail humain, 51, 273-288.

Bacher, F. (1989). Appendice méthodologique. In M. Reuchlin \& F. Bacher (éd.), Les différences individuelles dans le développement cognitif de l'enfant (pp. 232-293). Paris : Presses Universitaires de France.

Bacher, F. (1999). L'utilisation des modèles dans l'analyse des structures de covariance. L'Année psychologique, 99, 99-122.

Reuchlin, M. (1948). Contribution aux méthodes d'observation du comportement. L'Année psychologique, 49, 119-157. 
Reuchlin, M. (1951). Liaisons entre variables biométriques et variables socio-économiques. L’Année psychologique, 51, 267-289.

Reuchlin, M. (1953a). Plans expérimentaux et méthodologies en analyse factorielle. Binop, 9 , 79-86.

Reuchlin, M. (1953b). Utilisation en psychologie de certains plans d'expérience. L'Année psychologique, 53, 59-81.

Reuchlin, M. (1954). Hypothèses théoriques en psychologie quantitative. Journal de psychologie normale et pathologique, 47-51, 310-329.

Reuchlin, M. (1955a). Facteurs observés et facteurs théoriques en psychologie. In H. Laugier (éd.), L'Analyse factorielle et ses applications (pp. 187-208). Paris : CNRS.

Reuchlin, M. (1955b). Rapport de synthèse. In H. Laugier (éd.), L'Analyse factorielle et ses applications (pp. 395-407). Paris : CNRS.

Reuchlin, M., \& Bacher, F. (1957). Analyse factorielle de temps de réaction selon un plan P. Biotypologie, 18, 155-167.

Reuchlin, M. (1962a). Les Méthodes quantitatives en psychologie. Paris : Presses Universitaires de France.

Reuchlin, M. (1962b). La signification de la mesure en psychologie. In J.-M. Faverge, C. Flament, A. de Groot, L. Knops, M. Reuchlin, \& M. Yela (éd.), Les problemes de la mesure en psychologie (pp. 23-40). Paris : Presses Universitaires de France.

Reuchlin, M. (1963a). La mesure en psychologie. In P. Fraisse, \& J. Piaget (éd.), Traité de psychologie expérimentale, Tome 1 (pp. 185-221). Paris : Presses Universitaires de France.

Reuchlin, M. (1963b). La notion de dimension en psychologie différentielle. Psychologie française, $8(2), 80-95$.

Reuchlin, M. (1964). Méthodes d'analyse factorielle à l'usage des psychologues. Paris : Presses Universitaires de France.

Reuchlin, M. (1965a). Psychologie concrète et psychologie abstraite. Psychologie française, 10(4), 289-308.

Reuchlin, M. (1965b). Le caractère « comparable » de deux observations. Journal de psychologie normale et pathologique, 62(2), 179-200.

Reuchlin, M. (1967). Évolution, portée et limites de certains modèles utilisés en psychologie différentielle. In Actes du Colloque international du CNRS, Les Modèles et la formalisation du comportement (pp. 359-375). Paris : Éditions du CNRS.

Reuchlin, M. (1971). Formes vides et contenus informes en recherche pédagogique. Mathématiques et sciences humaines, 36, 59-77.

Reuchlin, M. (1976). Précis de statistique. Paris : Presses Universitaires de France.

Reuchlin, M. (1977). Épreuves d'hypothèses nulles et inférence fiduciaire en psychologie. Journal de psychologie normale et pathologique, 74(3), 277-292.

Reuchlin, M. (1978). Processus vicariants et différences individuelles. Journal de psychologie, 75(2), 133-145. 
Reuchlin, M. (1980). Théories en psychologie : explication et interprétation psychologiques. In M. Richelle \& X. Seron (dir.), L'explication en psychologie (pp. 237-260). Paris : Presses Universitaires de France.

Reuchlin, M. (1988). Un itinéraire de lecture : hasard et nécessité. Perspectives documentaires en sciences de l'éducation, 14, 19-27.

Reuchlin, M. (1990). Les niveaux d'observation et d'explication en psychologie : un débat épistémologique et institutionnel. In M. Reuchlin, F. Longeot, C. Marendaz, \& T. Ohlmann (dir.), Connaitre différemment (pp. 19-32). Nancy : Presses Universitaires.

Reuchlin, M. (1992). Introduction à la recherche en psychologie. Paris : Nathan.

Reuchlin, M. (1993). Problématiques et méthodologies structurales en psychologie. Revista Portuguesa de Psicologia, 29, 35-46.

Reuchlin, M. (1995). Totalités, éléments, structures en psychologie. Paris : Presses Universitaires de France.

Reuchlin, M. (1996). Une lecture de l'œuvre de Dominique Lépine. In J. Requin (éd.), Dominique Lépine, théoricien de la dualité (pp. 241-249). Paris : Éditions Commode.

Reuchlin, M. (1997, $1^{\text {re }}$ éd. 1969). La Psychologie différentielle. Paris : Presses Universitaires de France.

Reuchlin, M. (1998). Inférence statistique et inférence scientifique. Revue française de psychiatrie et de psychologie médicale, 18, 98-104.

Reuchlin, M. (1999). Évolution de la psychologie différentielle. Paris : Presses Universitaires de France. Reuchlin, M. (2003). Contributions à l'histoire des méthodes statistiques employées en psychologie. Psychologie et Histoire, 4, 1-60.

\section{NOTES}

1. Analyse en cluster reposant sur une procédure itérative (ramifying linkage method de Cattell) qui consiste à regrouper les traits en fonction de leurs intercorrélations.

2. . Dans les modèles unidimensionnels de réponse à l'item (Item Response Theory), les réponses individuelles aux items sont modélisées comme le résultat d'un processus stochastique dans lequel la probabilité de réponse à l'item est fonction du niveau en trait latent de l'individu et de la difficulté de l'item (modèle à 1 paramètre) ou de la difficulté et de la discrimination de l'item (modèle à 2 paramètres). Des extensions multidimensionnelles de ces modèles ont été aussi développées.

3. . L'analyse de dépendance permet de considérer simultanément plusieurs variables. Elle peut s'appliquer à des structures complexes manifestant ou non des effets d'interaction, comportant des variables continues ou discontinues, mais elle implique des calculs qui, à l'époque, pouvaient apparaître rebutants.

4. . Par exemple avec les logiciels LISREL, AMOS, EQS, Mplus ou lavaan (avec R). 


\section{RÉSUMÉS}

Reuchlin a exercé sa réflexion sur la méthodologie psychologique en discutant les fondements épistémologiques de cette méthodologie. De cette interaction entre options méthodologiques et réflexions épistémologiques ont émergé de riches enseignements dont on tente de dégager les aspects les plus caractéristiques. Le cheminement de pensée dans l'œuvre méthodologique de Reuchlin est illustré par ses réflexions sur la question de la mesure des différences individuelles, sur l'inadaptation des méthodes de description analytique des données à la complexité des problèmes posés en psychologie différentielle, sur l'intérêt d'une attitude structurale qui renouvelle le contenu des notions d'économie, de niveau d'analyse, de causalité et de fonction, sur l'utilité pour le psychologue des représentations structurales de la conduite dans lesquelles les variations d'une variable prise pour effet ne peuvent être expliquées qu'à l'échelle d'une structure de variables observables et / ou hypothétiques.

Reuchlin reflected on psychological methodology by discussing the epistemological foundations of this methodology. We try to identify the most characteristic aspects of the rich lessons that emerged from this meeting of methodological options and epistemological reflections. The progress of thought in the methodological work of Reuchlin is illustrated by reflections on the question of measuring individual differences, on the unsuitability of the data analytical description methods for the complexity of the problems studied in differential psychology, on the interest of a structural attitude which updates the content of the notions of economy, level of analysis, causality and function, and on the utility for the psychologist of structural representations of conduct, in which the variations of a variable taken for effect can only be explained by reference to a structure of observable and/or hypothetical variables.

\section{INDEX}

Keywords : Individual differences, usual life conditions, statistical inference, structural models, causality

Mots-clés : différences individuelles, conditions habituelles de vie, inférence statistique, modèles structuraux, causalité

\section{AUTEUR}

\section{JACQUES JUHEL}

est Professeur émérite de psychologie LP3C (EA 1285), Université Rennes-II Psychologie différentielle - Modélisation des différences individuelles, Contact : jacques.juhel@univrennes2.fr 